\title{
Intracranial hemorrhage in the era of the "direct" oral anticoagulants
}

\author{
Ileana Raluca Nistor, Leonida Gherasim \\ Neurology Department, Emergency University Hospital, Bucharest, Romania "Carol Davila" \\ University of Medicine and Pharmacy, Bucharest, Romania

\begin{abstract}
Intracranial hemorrhage $(\mathrm{ICH})$ is the most severe complication in patients treated with oral anticoagulants (OACs). Duration of anticoagulant therapy and poor anticoagulation control are important factors for hemorrhagic risk. Our purpose is to provide a review of meta-analyses regarding epidemiological data of intracranial hemorrhage, pathogenic conditions associated with IHC in patients treated with OACs, the role of clinical evaluation, data about prognostic and evolution of $\mathrm{IHC}$ and the general measures of the $\mathrm{IHC}$ treatment.
\end{abstract}

Keywords: intracranial hemorrhage, oral anticoagulants, hemorrhagic risk

\section{INTRODUCTION}

Intracranial hemorrhage $(\mathrm{ICH})$ is the most severe complication of oral anticoagulation, especially in patients with atrial fibrillation (AF) or venous thromboembolism (VTE). Duration of anticoagulant therapy and poor anticoagulation control are important factors for hemorrhagic risk.

ICH incidence in patients treated with AVK was reported in some randomized clinical studies between 0.36 and 0.85 /year, higher than $1.36 \%$ /year in AFI and 2.2\% in ACTVE-W trial (1). The use of new anticoagulants, currently named "direct" oral anticoagulants (DOACs) as dabigatran, rivaroxaban, apixaban and edoxaban reduced hemorrhagic risk with $57 \%$ versus warfarin ( $(R R 0,43 ; p<0,001)$ (2). IHC related to DOACs, though rare, are associated with very high mortality and morbidity rates, especially due to expanding hematoma. The mortality in a comparative study was $30-90 \%$ for both DOACs and warfarin (3). The recent use of anticoagulants antidotes increased the number of studies which have as objective intracranial and gastrointestinal bleedings during DOACs treatment.

\section{EPIDEMIOLOGICAL DATA}

The epidemiological data of $\mathrm{ICH}$ related to OACs (AVK, DOACs) comes from randomized clinical trials (DOACs vs warfarin), meta-analyses, and international collaborative studies. Overall, the incidence of ICH related to ACOs is different between AVK and DOACs; for AVK the IHC annual rate, in secondary prevention of $\mathrm{AF}$, ranged between 0.36 and 0.85 /year in some old studies and between 0.3 to $0.6 \%$ in more recent ones (4).

Ruff et al. meta-analysis, published in 2014, assessed the efficacy and safety of DOACs in nonvalvular atrial fibrillation and showed significant data. New oral anticoagulants reduced stroke or systemic embolic events by $19 \%$ compared with warfarin (RR 0.81, 95\% CI 0.73-0.91; p<0.0001), mainly driven by a reduction in hemorrhagic stroke (RR $0 \cdot 49 ; p<0 \cdot 0001)$. Taking into consideration that an equal number of patients received DOACs vs. warfarin (>29 000) the ICH incidence was lower in this group (approx. $1 / 2$ vs. warfarin). The lower number of ICH included intracerebral hematoma (the most severe ICH), subarachnoid and subdural bleeding. 
In Danish Registry of Medicinal Product Statistics IHC rate were $0.3 \% /$ year for dabigatran $150 \mathrm{mg}$ bid and $0.1 \%$ /year for dabigatran $110 \mathrm{mg}$ bid.

In summary IHC rate during DOACs treatment (doses from AF studies) is between 0.1 and $0.3 \%$ / year, approximately $1 / 2$ versus warfarin.

\section{CAUSES AND RISK FACTORS OF IHC RELATED TO OACS TREATMENT}

Pathogenic conditions associated with IHC in patients treated with OACs are similar with those found in general population. There are opinions that OACs are the trigger for IHC in coexisting vascular cerebral lesion and/or multiple risk factors.

Generally risk factors for IHC related to OACs are correlated with anticoagulant type and dose and patient-dependent factors.

The anticoagulant type and dose used (DOACs or AVK) are important first of all if respect the recommended dose from guidelines and clinical studies. Currently DOACs are preferred in secondary prevention of AF: DOACs reduced with $50 \%$ the IHC rate comparative with warfarin. In patients with severe renal failure (and some special condition) AVK are recommended.

Patient-dependent risk factors are many and have different importance.

Previous hemorrhagic stroke, secondary to large vessels injury is risk factor for repetitive IHC if the anticoagulant therapy is continued / re-started if is needed

Long term evolution or therapeutically uncontrolled systemic hypertension $(\mathrm{SH})$ is a major risk factor for IHC. The pathogenic process in hypertensive cerebrovascular vascular is small perforating cerebral arteries lipohyalinosis. IHC in $\mathrm{SH}-$ with and without OACs - have deep localization (putamen, internal capsule) and variable volume.

Asymptomatic cerebral amyloid angiopathy could be present in elderly. It is localized at capillaries and arterioles level and leads to microaneurysm formation. Pathogenic process is the deposition of $\beta$-amyloid in the walls leading to vascular fragility that increased risk of intracerebral bleeding, especially lobar ICH.

Previous cerebrovascular processes as arterial aneurysm, arteriovenous malformation, cavernous malformation, dural fistula are favorable factors for
IHC related to OAC.

Primary and secondary brain tumor could be complicated with intra-tumoral bleeding during the appropriate ACO treatment. The hematoma expansion is associated relatively fast with the worsening of neurological status.

"Cerebral microbleed" (CMB) - intracerebral microbleeds were considered in the last years a radiological biomarker, with IHC risk when ACO is associated. Cerebral microhemorrhages, or cerebral microbleeds, are small focal intracerebral hemorrhages, often only visible on susceptibility-sensitive MRI sequences; they appear as small, tiny, round dark-signal lesions localized at lobar level or deep. CMBs are tiny foci containing hemosiderin-laden macrophages secondary to self-limited microbleedings. In patients with ischemic stroke CMB were detected in almost $30 \%$ of the subjects (7).

CROMIS -2 study confirmed the meaning of CMBs detection in patients with AF and ischemic stroke treated with OACs (AVK, DOACs) (8). The symptomatic intracranial hemorrhage rate was 9.8 per 1000 patient-years in patients with cerebral microbleeds vs 2.6 per 1000 patient-years in those without cerebral microbleeds, so was a significant increased risk. The detection of CMB in patients with recent ischemic stroke and oral anticoagulant treatment is useful together with other bleeding scores (HAS-BLEED) for OACs treatment decision.

\section{CLINICAL EVALUATION OF IHC RELATED TO OACS TREATMENT}

Clinical evaluation has to determine the type of neurological signs and their gravity. Neurologic impairment, usually evolve faster (minutes or hours), starting with persistent and progressive cephalea, neurologic deficit, meningeal syndrome, up to major decreased level of consciousness. Clinical examination has to identify the underline disease for anticoagulant treatment (frequently secondary prevention of AF), the type and dose of DOACs, timing of last dose, comorbidities associated (especially renal impairment), other concomitant therapies and bleeding history.

Hemostasis evaluation would be useful for diagnosing IHC, together with standard evaluation. The 
coagulation tests selection is correlated with DOACs selection.

Thrombin time (TT) is the most sensitive test performed for dabigatran but is not yet standardized. Regardless the method used, TT is very sensitive, so a prolonged TT is correlated with low concentration of dabigatran (4). Normal value of TT in patients with ICH excludes a relevant concentration of dabigatran and not recommends the use of an antidote (idarucizumab). The dabigatran concentration could be quantified by ecarin clotting time (ECT).

The antifactor FXa assay is a useful test for quantifying the plasmatic levels of rivaroxaban, apixaban and edoxaban. An antidote might be be administered in IHC if anti FXa is more than $30 \mu \mathrm{g} /$ $\mathrm{ml}(9)$.

Activated Partial Thromboplastin Time (aPTT), Prothrombin Time (PT) and Thrombin Time (TT) are accessible and rapid tests while antifactor $\mathrm{Xa}$ assay and ECT results comes after hours, sometimes too late for taking a decision to neutralize DOACs.

Neuroimaging is essential for ICH diagnostic and treatment. Computed tomography identifies the stroke type - often hemorrhagic, but sometimes hemorrhagic transformation of ischemic stroke hematoma volume (ex. $<$ or $>20 \mathrm{ml}$ ) and localization, other coexisting cerebral lesions (amyloid angiopathy, leucoaraiosis etc. CMBs, a risk factor or a risk marker for $\mathrm{ICH}$, could be detected by magnetic resonance imaging (MRI) scan. MRI scan could suggest using these CMBs if DOACs treatment in $\mathrm{AF}$ increases the bleeding risk. A multivariate regression analysis showed that warfarin is independent associated with CMBs development (HR 10.75; $\mathrm{p}=0.03$ ) while DOACs are not correlated with CMBs occurrence.

\section{PROGNOSTIC AND EVOLUTION OF IHC}

The most probable evolution of "spontaneous" or OACs related IHC is hematoma expansion. The process could be explained by a pressure gradient (ruptured vessels/cerebral tissue); the gradient is higher in the first hours after injury and could decrease when during hematoma formation. The hipocoagulability degree done by OAC could contribute also. The worsening of neurological status, including the level of consciousness needs CT reevaluation for hematoma dynamic evaluation. The hematoma expansion is the main cause of mortality in $36-54 \%$ patients with ICH in the first hours after bleeding (2).

In patients without OAC the expansion of hematoma appears in only $30-40 \%$ in the first 4-6 hours after symptoms onset. The hematoma expansion rate is higher in prospective versus retrospective studies. (11).

The data regarding hematoma expansion and clinical evolution in OACs treatment are rare. Two collaborative studies showed similar data. Wilson et al. study included 13 registries published between 2014 and 2015, with 500 patients with ICH related to OACs (3). The second study from Germany, multicentric, integrative included 1,324 patients with ICH related to OAC, only 190 with DOACs (12). In both studies were followed the data about hematoma volume and expansion, ICH evolution at 90 days for AVK comparative with DOACs. The results showed no difference between hematoma characteristics and functional evolution in patients treated with DOACs vs AVK.

The data from ASPECT study are more optimistic. Were followed 2,245 patients with IHC, 227 (almost 10\%) with DOACs and 2,015 with warfarin. The number of IHC, the volume and the expan-

TABLE 1. IHC evolution related to DOACs vs warfarin

\begin{tabular}{|c|c|c|c|c|c|}
\hline & No pts with IHC & $\begin{array}{l}\text { Average volume IHC } \\
(\mathrm{ml})\end{array}$ & $\begin{array}{c}\text { Hematoma } \\
\text { expansion (\%) }\end{array}$ & $\begin{array}{c}\text { Mortality at } 90 \text { day } \\
\qquad(\%)\end{array}$ & $\begin{array}{c}\text { Unfavorable } \\
\text { evolution at } 90 \text { days } \\
(\%)\end{array}$ \\
\hline \multirow{4}{*}{ Wilson D study } & 500 & & & & \\
\hline & $97 \mathrm{DOAC}$ & 10.6 & 40 & 35 & \\
\hline & 403 AVK & 14.4 & 34 & 35 & \\
\hline & & $\mathrm{P}=0.78$ & $\mathrm{P}=0.45$ & & \\
\hline \multirow{4}{*}{$\begin{array}{l}\text { Gerner ST study } \\
\text { (2019) }\end{array}$} & 1324 & & & & \\
\hline & $190 \mathrm{DOAC}$ & 14.7 & 33.6 & & 70.4 \\
\hline & 1134 AVK & 16.4 & 34.2 & & 63.4 \\
\hline & & $\mathrm{P}=0.33$ & $\mathrm{P}=0.89$ & & $\mathrm{P}=0.79$ \\
\hline
\end{tabular}


sion of hematoma and even the mortality were lower with DOACs vs AVK (13).

The mortality in "spontaneous" or OAC related IHC is high. In warfarin era $30-85 \%$ of deaths were due to intracerebral hemorrhage. In patients with IHC and AVK the mortality was evaluated at $>50 \%$ especially if INR was over therapeutic range (14).

The usage of DOACs in secondary prevention of AF proved a significant decrease in IHC rate vs AVK (RR 0.43; $<<0.01$ ). The low absolute number of IHC related to DOACs was associated with a decrease in mortality due to IHC. In the large trials with DOACs vs warfarin the mortality due to ICH is lower but without statistical significance: RE-LY $36 \%$ AVK vs $35 \%$ dabigatran; ROCKET-AF $50 \%$ AVK vs $48 \%$ rivaroxaban; ARISTOTLE $42.6 \%$ AVK vs $48 \%$ apixaban $(15,16,17)$. In the German study, multicentric, prospective, published in 2015 the mortality rate was $28 \%$ and favorable evolution in $25 \%$ of IHC patients (18). The favorable evolution of IHC related to DOACs was recorded for $28 \%$ of the patients (score $m R S \leq 2)(19)$.

The use of antidotes that reversal the activity of DOACs might conduct to a better evolution in IHC.

The treatment of IHC related to OACs, especially DOACs is a major emergency due to bleeding localization (intracerebral) and possible hematoma extension. It is mandatory to stop the bleeding and IHC evolution using active agents for reversal quickly the hipocoagulability status.

The treatment could be initiated after overall clinical examination, neurological evaluation - including neuroimaging - to establish the type of anticoagulant used by patient, dose, timing of last dose (in hours) and of course the differential diagnose for other IHC etiologies.

In summary the treatment includes: 1. general measures common for all types of IHC; 2 . the possibility to use and the indication for administration of a specific reversal agents; 3 . hemostatic treatment with proven effectiveness; 4. neurosurgery approach - the last option in life threating IHC (20).

The general measures are: the DOACs should be stopped; other drugs that can affect bleeding (ex antiplatelet agents) should also be noted; the blood pressure control, with a target $<140 \mathrm{mmHg}$ (21); fluids to facilitate the metabolism and renal excretion of DOACs; blood transfusion for severe post hemorrhagic anemia $(\mathrm{Hb}<8 \mathrm{~g} / \mathrm{dL})$ especially if a gastrointestinal bleeding coexist.

The usage of specific treatment in IHC related to DOACs is dependent on the type of anticoagulant, dose, timing of last dose (hour) and the anticoagulant activity evaluation. Generally for DOACs $t 1 / 2$ is about 12 hours and peak time 1-3 hours. TT and aPTT (eventually ECT) can be used to assess anticoagulant activity of dabigatran and anti FXa assay for FXa inhibitors. These data offer fast but partial information about anticoagulant status in patients treated with DOACs (22).

Specific reversal agents or the emergency treatment of IHC related to DOACs are Idarucizumab (for dabigatran), Andexat alfa (for rivaroxaban, apixaban, endoxaban) and Ciraparantag (for both types of DOAC). Prothrombin complex concentrate (PCC) is a non-specific hemostatic agent that could be used in major bleedings related to DOACs.

Idarucizumab is a humanized monoclonal antibody fragment $(\mathrm{Fab})$ indicated in patients treated with dabigatran when reversal of the anticoagulant effects are needed which binds dabigatran reversibly with 350-fold higher affinity than thrombin and forms a 1:1 stoichiometric complex rapidly cleared by the kidney with captured dabigatran (23).

The idarucizumab efficacy was proved in REVERSE-AD study (Reversal Effects of Idarucizumab on Active Dabigatran) that enrolled 90 patients with major bleeding related to dabigatran (intracerebral, gastrointestinal, surgical), 18 with ICH (24). The study was recently completed with 504 patients treated with dabigatran needed to reverse rapidly the anticoagulant effect. The patients received two bolus infusions of $2.5 \mathrm{~g}$ of idarucizumab, no more than 15 minutes apart. After 4 hours the reversal of anticoagulant effect was $100 \%$ for TT, aPTT and ECT (4). The mortality in those 18 patients with $\mathrm{ICH}$ receiving idarucizumab was $22.5 \%$ instead that in RE-LY the mortality in patients with ICH and dabigatran was $41 \%$.

Currently idarucizumab could be indicated in emergency in patients with severe bleedings $(\mathrm{ICH}$, digestive hemorrhage) but also in emergency surgery when major bleeding is not under control. After idarucizumab (like other reversal agents for DOACs) thrombotic events could appear in almost $10 \%$ of treated patients (24). 
Andexanat alfa is a recombinant variant of human FXa that acts competitive with native FXa. Andexanat alfa protein acts as a decoy for the direct oral FXa inhibitors rivaroxaban, apixaban and endoxaban in the blood up to their excretion. The decrease in FXa activity is very fast (minutes) (25). Andexanat alfa reverses and anticoagulant effects of unfractioned heparin, low molecular weight heparin and fondaparinux competitive with FXa and thrombin (26).

The first results about treatment with adenaxet in major bleedings related to rivaroxaban, apixaban and endoxaban were published in ANNEXA-4 study (26). In the study were enrolled 67 patients with major bleedings (IHC, gastrointestinal) who received a FXa anticoagulant in the last 7-8 hours before bleeding. Andexanat alfa was administered as a $400 \mathrm{mg}$ intravenous bolus followed by a continuous infusion of $480 \mathrm{mg}$ in 120 minutes (apixaban, rivaroxaban) or as a $800 \mathrm{mg}$ intravenous bolus followed by a continuous infusion of $960 \mathrm{mg}$ in 120 minutes (enoxaparine, edoxaban). The endpoints were the changes in anti-factor Xa activity and the percentage of patients with excellent or good hemostatic efficacy at 12 hours after the end of the infusion. The adenexat bolus decreased the median anti-factor Xa activity in treated patients: $89 \%$ for rivaroxaban and $93 \%$ for apixaban. Excellent or good hemostasis occurred in $79 \%$ patients. There were $18 \%$ patients with a thrombotic event during the 90-day follow-up period.

The full study report of ANNEXA-4 was recently published (27). In the study were evaluated 352 patients who had acute major bleeding within 18 hours after administration of a factor Xa inhibitor with bleeding intracranial (in 227 patients [64\%]) or gastrointestinal (in 90 patients [26\%]). In patients who had received apixaban and rivaroxaban the decrease in median anti-factor Xa activity was similar $(92 \%)$. Excellent or good hemostasis occurred in $82 \%$ patients (204 of 240), within 30 days, death occurred in $14 \%$ patients and a thrombotic event in 10\%. Thrombotic events (myocardial infarction, ischemic stroke and deep vein thrombosis) appeared within 5-30 days after andexanet and almost $25 \%$ of the patients restart anticoagulant treatment after 30 days.

In May 2018, andexanet alfa received its first global approval in the USA for use in patients treat- ed with rivaroxaban and apixaban, when reversal of anticoagulant effects is required in life-threatening or uncontrolled bleeding.

Ciraparantag (PER977) is a small synthetic water-soluble new molecular entity studied for reversal anticoagulant effect of DOACs.

Ciraparantag binds specific to endoxaban, apixaban, rivaroxaban but also heparin and low molecular weight heparin removing these drugs from their intended target and allowing rapid re-establishment of normal blood coagulation (4). In phase I study on healthy volunteers PER was administered oral, sc or iv. The effect persisted for 24 hours, and there was no increase in the levels of D-dimer or thrombin-antithrombin complexes suggestive of a procoagulant state. (28). Ciraparantag is an under evaluation drug, phase III studies have not been initiated so it is not approved for treatment.

In the rapid reversal of hemorrhagic effect of DOACs were tested more products which included more coagulation factors: active and inactive prothrombin complex concentrate (PCC). Limited research showed that PCC (four factors) could reverse quickly and complete the anticoagulant effect of dabigatran and rivaroxaban $(29,30)$.

In some studies recently published $(2017,2018)$ PCC was used in IHC related to FXa inhibitors. In a retrospective study that included 146 patients, $71 \%$ of them received PCC. The hematoma expansion ( $\geq 33 \%$ vs baseline) was recorded in $34 \%$ patients (31). In a prospective study (184 patients) an effective hemostasis was recorded in $64 \%$ patients treated with PCC (32) with $32 \%$ mortality within 30 days, 3 cases by thrombotic events. Thromboembolic events were registered between 10 to $14 \%$ in patients with severe bleedings after PCC treatment, but also with other DOACs reversal agents.

Today, the use of PCC for bleedings related to DOACs has no evidence for efficacy and safety so there is not approved by FDA in this indication.

Surgical treatment of IHC could be indicated especially for hematoma expansion and in case of lack of efficacy of maximal medical treatment. In a Japanese study the rates of surgery for hematoma removal were significantly lower in the patients with DOAC-associated ICH (DOAC, 2.3\%; Warfarin, 9.7\%; $\mathrm{P}=0.019$ ) (33).

Before specific reversal agents [Idarucizumab (for dabigatran), Andexat alfa (for rivaroxaban, 
apixaban, endoxaban)] use in DOACs-associated major bleedings were indicated active charcoal (mainly in the first hours after DOACs administration) and hemodialysis (for dabigatran excretion). The hemodialysis could be used in renal failure patients receiving dabigatran inadequate doses (4).

Restarting anticoagulant therapy after intracranial hemorrhage is a difficult decision that must take in consideration the IHC recurrence risk, timing of medication resumption, the right type of anticoagulant, the thromboembolic risk without anticoagulation.

The data about recurrence risk are some under development and some contradictory. In patients with acute ischemic stroke and AF, DOACs treatment is associated with 5\% composite rate of embolic ischemic event recurrence and severe bleeding within 90 days (11). In patients without anticoagulant treatment and previous IHC the annual risk rate of recurrence is between 0 and $8.6 \%$. In those with resumption of anticoagulation annual risk is between 2.5 and $8 \%$. A recently meta-analysis including 8 studies and 5,303 ICH showed that the risk of recurrent $\mathrm{ICH}$ is similar with or without reinstatement of anticoagulation (35).

Before to decide restarting of anticoagulant therapy and the right timing for this is suitable to perform a CT or a MRI to confirm both IHC resolution and risk factors presence; together with risk factors for thromboembolism recurrence evaluation.

The anticoagulation resumption is important in patients with mechanical prosthetic valve, AF and
$\mathrm{CHA}_{2} \mathrm{DG}_{2}-$ VASC score $\geq 3-4$ or high risk VTE (6). In these cases the reinitiation of anticoagulation significantly decreases the thromboembolic complication: HR 0.34, 95\% CI (35).

The reinitiation time of anticoagulation is different between studies, from 7 to 30 days.

In a cohort Danish study $(n=1,725)$ the anticoagulation resumption time was between 2 to 10 weeks after ICH (36); when OAC were started after 4 weeks post ICH the recurrence risk decreased with 42 to $59 \%$. In clinical practice the OAC restarting depends on individual clinical status (6) regarding hemorrhagic and thromboembolic risk; for example anticoagulant resumption in patients with mechanic prosthesis could be reduced at 2 weeks especially if intracerebral bleeding was mild and the cause was solved.

The type of OACs used and preferred is the physician choice. The decrease in IHC risk by using DOACs (proven in studies) and even the lower risk of DOACs vs AVK are favorable argues for anticoagulation - when needed - with DOACs. The opportunity to use specific anticoagulant reversal agents (idarucizumab, andexanet alfa) with beneficial effects on IHC incidence might be additional arguments to select an $\mathrm{OAC}$ to reinitiate anticoagulation after IHC.

To reduce the recurrence risk of IHC when anticoagulation is contraindicated could be taken extreme measures as left atrial appendage, AF ablation.

Conflict of interest: none declared Financial support: none declared

\section{REFERENCES}

1. You JJ, Singer DE, Howard QA et al. Antithrombotic therapy for atrial fibrillation. Antithrombotic Therapy and Prevention of thrombosis 9th ed. Chest 2012, 141 (2), Suppl e 531 S-e 575 S.

2. Ruff CT, Giuliano RP, Braunwald $L$ et al. Comparison of the efficacy and safety of new oral anticoagulants with warfarin in patients with atrial fibrillation: A meta-analysis of randomized trials. Lancet 2014, 383:955-62

3. Wilson D, Seiffpe DJ, Traenka C et al. Outcome of intracerebral hemorrhage associated with different oral anticoagulants. Neurology 2017, 88: 1693-1700.

4. Steiner T, Weitz JI, Veltkamp R. Anticoagulant-Associated Intracranial Hemorrhage in the Era of Reversal Agents. Stroke. 2017 May;48(5):1432-1437.

5. Larsen TB, Rasmussen LH, Skpeth F et al. Efficacy and safety of dabigatran etexilate and warfarin in "real world" patients with atrial fibrillation: A prospective nationwide cohort study. J Am Coll Cardiol, 2013, 61:2264-73.

6. Yan-guang Li, Lip GYH. Anticoagulation Resumption After Intracerebral Hemorrhage. Curr Atheroscler Rep. 2018; 20(7):32.

7. Charidimou A, Karayiannis C, Song TJ et al. Brain microbleeds, anticoagulation, and hemorrhage risk: Meta-analysis in stroke patients with AF. Neurology. 2017 Dec 5;89(23):2317-2326.

8. Wilson D, Ambler G, Shakeshaft C et al. on behalf of CROMIS-2 collaborators. Cerebral microbleeds and intracranial haemorrhage risk in patients anticoagulated for atrial fibrillation after acute ischaemic stroke or transient ischaemic attack (CROMIS-2): A multicentre observational cohort study. Lancet Neurol. 2018 Jun;17(6):539-547.

9. Lu G, DeGuzman FR, Hollenbach SJ et al. A specific antidote for reversal of anticoagulation by direct and indirect inhibitors of coagulation factor Xa. Nat Med. 2013 Apr;19(4):446-51.

10. Greenberg SM, Vernooij MW, Cordonnier $C$ et al. Cerebral microbleeds: A guide to detection and interpretation. Lancet Neurol. 2009 Feb;8(2):165-74

11. Paciaroni M, Agnelli G, Falocci $\mathrm{N}$ et al. Early Recurrence and Major Bleeding in Patients With Acute Ischemic Stroke and Atrial Fibrillation Treated With Non-Vitamin-K Oral Anticoagulants (RAF-NOACs) Study. J Am Heart Assoc. 2017 Nov 29;6(12). 